\title{
Treatment of A Late Presenting Displaced Radial Neck Fracture in A 10 Years-old Girl
}

\author{
Thomas G. Papageorgiou a, Nikolaos E. Panos ${ }^{\mathrm{a}}$, Ioannis P. Gigis ${ }^{\mathrm{a}, \mathrm{b}}$, \\ Efthimios P. Samoladas ${ }^{\mathrm{a}}$, Theodoros A. Beslikas ${ }^{\mathrm{a}}$, \\ Ioannis E. Christoforidis ${ }^{\mathrm{a}}$
}

\begin{abstract}
Pediatric radial neck fractures are relatively common injuries resulting from a fall onto an outstretched arm. Late diagnosis and treatment of pediatric radial neck fractures are extremely rare. We report a case of a late-presenting displaced radial neck fracture in a 10-year-old girl, type III with translocation of the radial head on the neck. The child was seen in our department 120 days after the injury and was treated with open reduction and internal fixation with $\mathrm{K}$-wire. The arm was immobilized and the K-wire was removed 6 weeks after insertion and the patient underwent a step-wise rehabilitation programme. Three months later, the patient was symptom free, had a normal carrying angle as well as an excellent range of movement with full pronation and supination. Early recognition, proper management and physical therapy led to complete recovery and full functional movement of the elbow.
\end{abstract}

Keywords: Pediatric radial neck fracture; Internal Fixation; Avascular necrosis

\section{Introduction}

Fractures of the radial neck account for slightly more than $1 \%$ of all children fractures [1]. In $90 \%$ of proximal radial fractures, the fracture line involves either the physis or the neck $[1,2]$.

In children, the cartilaginous radial head is resistant to fracture, and children are more likely to sustain fractures of

Manuscript accepted for publication July 29, 2011

a2nd Orthopaedic Department, “G. Gennimatas” General Hospital,

Aristotle University of Thessaloniki, Greece

${ }^{\mathrm{b}}$ Corresponding author: Ioannis P. Gigis, Profiti Ilia 13, Pylea, 55535,

Thessaloniki, Greece. Email: jgigis@otenet.gr

doi:10.4021/jmc267w the radial neck than fractures of the head [2]. Radial neck fractures rarely produce obvious clinical deformity. In fact, the fracture may not be evident on initial radiographs and may be noticed only when callous begins to be seen radiographically after 7 to 14 days. After the diagnosis has been made, results are best if the treatment is initiated early and it has been argued that closed reduction usually leads to satisfactory results.

Older children have been found to have a worse prognosis [3]. Some authors have found open reduction is associated with only a fair or poor result.

In younger children less than 10 years of age, $10^{\circ}$ or less of residual neck angulation will correct with growth and up to $30^{\circ}$ of residual neck angulation can accepted [1, 4]. Greater than $30^{\circ}$ of neck angulation requires closed manipulative reduction or percutaneous wiring to manipulate the fracture [4]. In older children of 10 years or more, the outcome is poor if there is neck angulation of greater than $30^{\circ}$ or a translocation of the fracture segment greater than 3 $\mathrm{mm}[3,4]$. An inability to reduce and angulation of less than $45^{\circ}$ may require open reduction and internal fixation $[4,5]$. An inability to pronate and supinate the forearm more than $60^{\circ}$ is a sign that the reduction is inadequate.

\section{Case Report}

A 10 years-old right-handed girl fell onto an outstretched left arm with the elbow in extension and valgus. The patient instantly suffered pain and swelling over the lateral aspect of the left elbow with reduced range of movements at that site.

She attended a local minor injuries unit complaining of swelling and tenderness over her lateral elbow. The initial $\mathrm{X}$-rays were suspicious of a proximal radial injury. She was discharged with an arm sling.

The child was submitted in our out-patients department 120 days after the injury with ongoing swelling and tenderness over the left proximal radius. Elbow flexion and extension were reduced but more significant was the reduction in pronation and supination.

An x-ray (Fig. 1) as well as a 3D reconstruction CT were carried out confirming a completely displaced fracture Type 


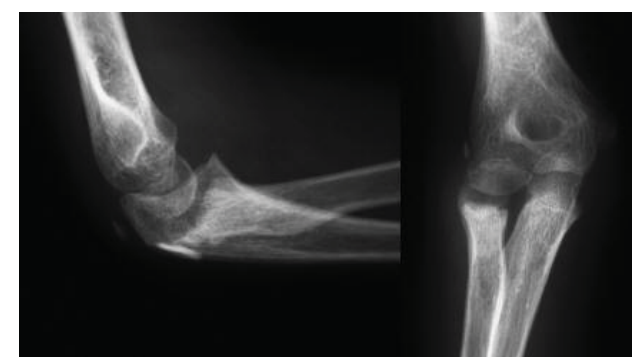

Figure 1. Lateral and Antero-posterior x-rays demonstrating a completely displaced fracture with translocation of the radial head on the neck.

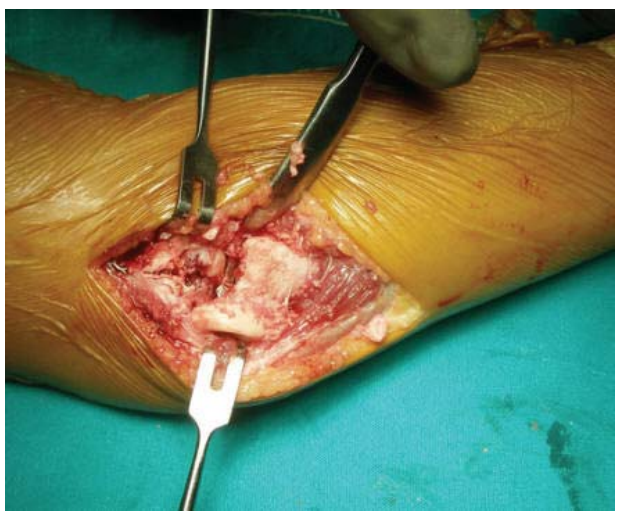

Figure 2. The displaced radial head during the open reduction.

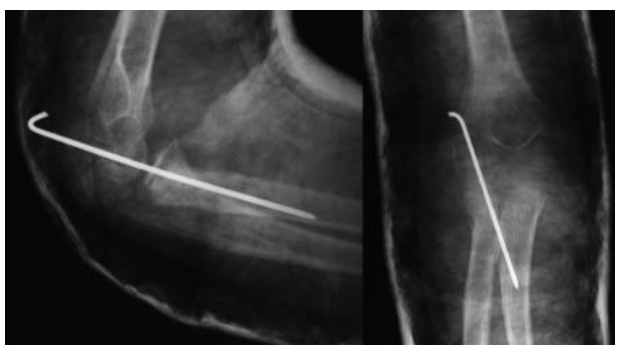

Figure 3. Post-operative $x$-rays showing internal fixation of the radial head fracture with the use of a K-wire.

III $>60^{\circ}$ (O’Brien PI) with translocation of the radial head on the neck. The child was treated with open reduction (Fig. 2) and internal fixation with a K-wire through a postero- lateral approach with as little dissection as possible to manipulate and stabilise the radial neck fragment, with satisfactory postoperative check radiographs confirming adequate reduction and fixation of fracture segments (Fig. 3).

The epiphysis appeared stabilised. The K-wire was removed 6 weeks after insertion (Fig. 4) and the patient underwent a step-wise rehabilitation programme. Three months after presentation, the patient was symptom free, had a normal carrying angle and had excellent range of movement at the elbow with full pronation and supination. Check radio-

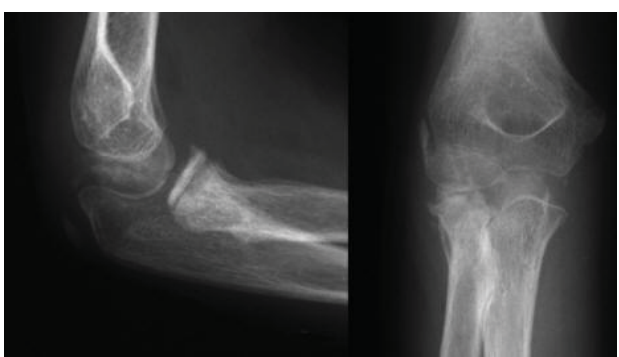

Figure 4. X-rays carried out 6 weeks post-operative after the removal of the K-wire.

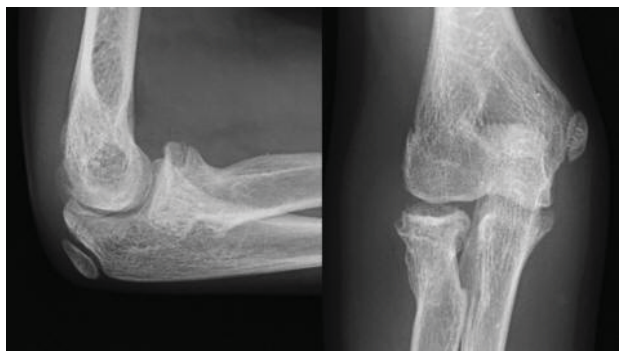

Figure 5. X-rays 2 years post-operative showing completed healing of the fracture as well as correct alignment of the radial neck.

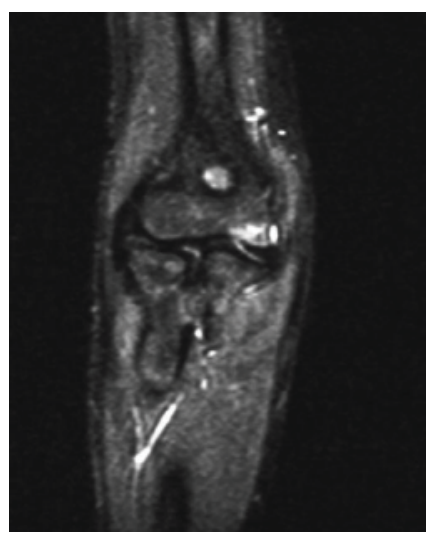

Figure 6. MRI at 2 years port-operative demonstrating a viable radial head with no signs of avascular necrosis.

graphs showed the cortices at the radial neck fracture site had healed in correct alignment with a normal appearance of the corresponding epiphysis. X-rays (Fig. 5) and MRI (Fig. 6) of her right elbow at 2 years post operatively with gadolium revealed that the radial head is still viable with no signs of avascular necrosis. The patient was successfully discharged from our care.

\section{Discussion}

Radial neck fractures often occur from a fall on an extended and supinated outstretched hand. Like all falls with this 
mechanism, the energy is dissipated through the upper limb and in this case it is transmitted through the shaft of the radius with the capitellum being driven against the radial head [1]. Subsequent head on neck angulation is depending on the position of the radius with full supination leading to lateral displacement and neutral forearm positioning leading to posterior displacement. Radiological signs include a posterior fat-pad sign and one should consider radiocapitellar views [6]. Treatment of radial neck fractures is dependent on the time of presentation to the surgeon and the level of displacement of the radial head on the radial neck [3]. Minimally displaced fractures may be treated by immobilisation whereas moderately displaced fractures may require reduction under anaesthetic. The aim must be to achieve less than $30^{\circ}$ of angulation and correction of any translocation such that the epiphysis is normally aligned. Indeed authors have found good outcome when conservatively managing these fractures if the angulation is less than $30^{\circ}$ [5]. Another important mechanical factor is the restoration of the radial neck length. A recent study [7] showed lengthening or shortening of more than $2.5 \mathrm{~mm}$ leads to axial understuffing or overstuffing of the radiohumeral joint and significantly changes elbow kinematics. Steinmann pins have also been used to percutaneously reduce and fixate angulated and displaced radial neck fracture but this method appears to be limited by severe radial head displacement, which leads to a moderate rate of fair and poor results [4]. With severe head displacement, some authors have found, open reduction and temporary transarticular K-wire internal fixation leads to a high rate of poor results [8]. Additionally, open reduction and internal fixation of these fractures has been widely reported to lead to intra-articular calcification, avascular necrosis, and joint stiffness [8-10]. This case highlights that open reduction and internal fixation with one $\mathrm{K}$-wire is a safe and effective treatment of displaced radial neck fractures even when the presentation is delayed after injury. It also shows that avascular necrosis of the radial head didn't occurred although the radial head remained displaced for 4 months.

\section{Conclusion}

It is the first case in the literature, which demonstrates that in young patients the radial head can be viable with proper surgical technique even in a delayed management. The possible explanation is that during surgical dissection we preserved the medial attachment of the radial head and we used limited hardware.

\section{Conflicts of Interest}

None financial remuneration has been received from the authors or any member of their family. This study was approved by the Hospital Ethical Committee (Study No: 41).

\section{References}

1. Chambers HG. Fractures of the proximal radius and ulna. In: Kasser JR, Beatty JH, editors. Rockwood and Wilkins' fractures in children. 5th ed. Philadelphia: Lippincott Williams and Wilkins; 2001. p. 483-528 (ISBN No. 978-0781725095).

2. Malmvik J, Herbertsson P, Josefsson PO, Hasserius R, Besjakov J, Karlsson MK. Fracture of the radial head and neck of Mason types II and III during growth: a 1425 year follow-up. J Pediatr Orthop B. 2003;12(1):6368.

3. Tibone JE, Stoltz M. Fractures of the radial head and neck in children. J Bone Joint Surg Am. 1981;63(1):100106.

4. Rodriguez Merchan EC. Percutaneous reduction of displaced radial neck fractures in children. J Trauma. 1994;37(5):812-814.

5. Vahvanen V, Gripenberg L. Fracture of the radial neck in children. A long-term follow-up study of 43 cases. Acta Orthop Scand. 1978;49(1):32-38.

6. Beaty JH, Kasser JR. The elbow region: general concepts in the pediatric patient. In: Kasser JR, Beaty JH, editors. Rockwood and Wilkins' fractures in children. 5th ed. Philadelphia: Lippincott Williams and Wilkins; 2001. p. 563-575 (ISBN No. 978-0781725095).

7. Van Glabbeek F, van Riet RP, Baumfeld JA, Neale PG, O’Driscoll SW, Morrey BF, An KN. The kinematic importance of radial neck length in radial head replacement. Med Eng Phys. 2005;27(4):336-342.

8. D’Souza S, Vaishya R, Klenerman L. Management of radial neck fractures in children: a retrospective analysis of one hundred patients. J Pediatr Orthop. 1993;13(2):232238.

9. Rodriguez Merchan EC. Displaced fractures of the head and neck of the radius in children: open reduction and temporary transarticular internal fixation. Orthopedics. 1991;14(6):697-700.

10. Metaizeau JP. Reduction and osteosynthesis of radial neck fractures in children by centromedullary pinning. Injury. 2005;36 Suppl 1:A75-77. 